\title{
A Blind DWT-SCHUR Based Digital Video Watermarking Technique
}

\author{
Lama Rajab1, Tahani Al-Khatib1, Ali Al-Haj2 \\ ${ }^{1}$ Computer Information Systems, The University of Jordan, Amman, Jordan \\ ${ }^{2}$ Princess Sumaya University for Technology, Amman, Jordan \\ Email: Lama.rajab@ju.edu.jo, tahani.khatib@ju.edu.jo, ali@psut.edu.jo
}

Received 26 March 2015; accepted 23 April 2015; published 24 April 2015

Copyright (C) 2015 by authors and Scientific Research Publishing Inc.

This work is licensed under the Creative Commons Attribution International License (CC BY).

http://creativecommons.org/licenses/by/4.0/

(c) (i) Open Access

\begin{abstract}
Digital watermarking is one of the most powerful tools used in ownership and copyrights protection in digital media. This paper presents a blind digital video watermarking technique based on a combination scheme between the Discrete Wavelet transform in (DWT) and the real Schur Decomposition. The scheme starts with applying two-level DWT to the video scene followed by Schur decomposition in which the binary watermark bits are embedded in the resultant block upper triangular matrix. The proposed technique shows high efficiency due to the use of Schur decomposition which requires fewer computations compared to other transforms. The imperceptibility of the scheme is also very high due to the use of DWT transform; therefore, no visual distortion is noticed in the watermarked video after embedding. Furthermore, the technique proves to be robust against set of standard attacks like: Gaussian, salt and pepper and rotation and some video attacks such as: frame dropping, cropping and averaging. Both capacity and blindness features are also considered and achieved in this technique.
\end{abstract}

\section{Keywords}

Video Watermarking, Schur Decomposition, Discrete Wavelet Transform (DWT)

\section{Introduction}

As Digital videos and images are remarkably expanding over the internet due to the development of blogs, video sharing websites and social network applications, it becomes necessary to protect copyrights of the digital contents to guarantee the ownership identification. Digital watermarking in multimedia has become the most efficient technology used in securing digital data. In practice, the watermarking technique embeds a small copyright message, called a watermark, inside the digital media content to identify the ownership. 
Effective techniques in watermarking should consider both imperceptivity and robustness of the algorithm. Imperceptibility refers to the watermark perceptual transparency [1], while robustness indicates the ability of extracting the embedded watermark from the digital media under intentional or unintentional attacks such as degradation, cropping, filtering or additive noise [1] [2]. Capacity and blindness of the watermarking techniques are two additional issues to be considered as well. Capacity, also referred as data Payload, includes methods that make it possible to embed the majority of information. Blindness of the algorithms implies that the watermarking extraction does not need the existence of the original image [3].

In literature, many watermarking algorithms have been proposed for video, video frames and images. In general, Digital watermarking techniques are classified into two categories: spatial domain watermarking, as in [4]-[6], and transform domain watermarking, such as in [7]-[10]. In spatial domain methods, watermarking is performed by modifying the pixels values in the host image. However, in frequency domain methods, the watermark is embedded into transformed coefficients of the image. Frequency domain methods are found to be more robust and complex than spatial domain ones; also they are visually more convenient to human and have higher imperceptibility and capacity [11].

All kinds of digital media may be protected in order to guarantee ownership and copyrights identification, and hence watermarking can be applied on digital videos, audios, images or documents. In the case of digital videos, watermarking may be applied on both compressed and uncompressed videos. However, applying watermarking on uncompressed videos is much safer than compressed ones because video frames are considered as consecutive and equally spaced still; and hence, watermark will be embedded without any data loss risk. In contrast, watermarked compressed video such as MPEG-compressed bit stream can be converted to another compression standard which may result in modifying or losing the embedded watermark data [12].

There are many researches on transforming domain watermarking for audio, images, compressed and uncompressed videos. Various proposed algorithms made use of popular transformations such as SVD, DWT, or DCT. Yet, many algorithms combined some of these transformations to form hybrid approaches in order to obtain better embedding and extraction results. In some new researches, SCHUR transform was studied and used in watermarking techniques. Using Schur transform in watermarking embedding and extraction had proved to be a promising area that may compete with other transform domains and make good results for robustness and precision in watermarking.

In [13], authors presented a non blind hybrid watermarking scheme using SCHUR and SVD transforms. They used Schur in embedding and extraction steps after decomposing the image into $8 \times 8$ blocks followed by applying SVD on the largest Eigen values. However, in [14], authors proposed a non blind hybrid technique using SCHUR and Cellular Automata Transform (CAT), and made use of the flexibility of CAT domain and the efficiency of the Schur transform. While in [15], a blind image watermarking method based on Schur transform was proposed. The image is transformed into Schur transform and then analyzed to find a non-sensitive zone where embedding procedure take place. On the other hand, the proposed algorithm in [16] embeds the watermark in two decomposition of SCHUR transform, which are the D matrix and the U matrix, the technique improves the robustness of the algorithm since the same watermark information is stored twice in two places.

In this paper, a robust, blind digital video watermarking technique is proposed. The proposed technique combined the Schur decomposition with the DWT transform to produce an efficient technique with high robustness and imperceptibility. The rest of this paper is organized as follows: The Discrete Wavelet transform and the Schur decomposition is described in Section 2. In Section 3 the proposed watermarking technique is discussed in details. In Section 4 the experimental results are discussed and Section 6 concludes the proposed technique.

\section{Materials and Methods}

\subsection{Discrete Wavelet Transform (DWT)}

Discrete Wavelet Transform is the multi resolution description of an image, where the decoding can be processed sequentially from a low resolution to the higher resolution [17]. The discrete wavelet transform is based on small waves, called wavelets, of varying frequency and limited duration. Such wavelet transform provides both frequency and spatial description of an image [18]. The Discrete Wavelet Transform DWT splits the signal into high and low frequency parts. The high frequency part contains information about the edge components, while the low frequency part is split again into high and low frequency parts. The high frequency components are usually used for watermarking since the human eye is less sensitive to changes in edges [19]. The discrete 
wavelet transform decomposes the input image hierarchically to four frequency districts one low (LL) and the other three districts are high: LH, HL, HH as shown in Figure 1.

A two dimensional image can be decomposed by DWT by more than one level. In the proposed technique we applied 2 level DWT, and then we used the High level (HL) band in the second level. Each sub-band is a matrix of DWT coefficients at a specific resolution. Figure 2 shows the sub-bands produced by the 2-level DWT decomposition.

\subsection{Schur Decomposition}

The Schur decomposition or Schur triangulation is an important mathematical tool in linear algebra used in metrics analysis, which was named after Issai Schur. In fact, SCHUR decomposition represents a major intermediate step in SVD decomposition [20]; and it to be an efficient mathematical tool since it requires about $\mathrm{n}^{3}$ flops for an $\mathrm{n} * \mathrm{n}$ matrix; while SVD decomposition requires $11 \mathrm{n}^{3}$ flops. Which means that the number of computations performed in SCHUR is less than one third of those performed in SVD decomposition [21].

There are two versions of Schur decomposition transform: complex Schur and real Schur, [22]. The Real Schur decomposition version of a real square matrix A results in two matrices: $\mathrm{U}$ and $\mathrm{S}$ as shown in Equation (1):

$$
\operatorname{Schur}(\mathrm{A})=\left(\mathrm{U} \times \mathrm{S} \times \mathrm{U}^{\mathrm{T}}\right)
$$

where $\mathrm{S}$ is the block upper triangular matrix called the real Schur form and $\mathrm{U}$ is a unitary orthogonal matrix. $\mathrm{U}^{\mathrm{T}}$ indicates the conjugate transpose of $\mathrm{U}$. The following example illustrates the Schur decomposition applied on a $3 \times 3$ matrix A.

$$
A=\left[\begin{array}{lll}
160 & 160 & 159 \\
160 & 159 & 159 \\
161 & 160 & 158
\end{array}\right]
$$

Applying real Schur decomposition on matrix A will produce both U and S matrices as follows:

$$
\begin{gathered}
U=\left[\begin{array}{ccc}
0.5778 & 0.4077 & -0.7071 \\
0.5765 & -0.8171 & -0.0000 \\
0.5778 & 0.4077 & 0.7071
\end{array}\right] \\
S=\left[\begin{array}{ccc}
478.6664 & -0.7071 & -2.0418 \\
0 & -0.6664 & -0.5753 \\
0 & 0 & -1.0000
\end{array}\right]
\end{gathered}
$$

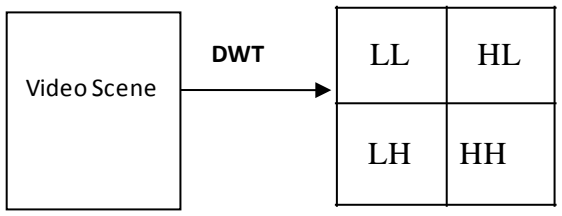

Figure 1. Frame’s 2-level DWT sub-bands.

\begin{tabular}{|c|c|c|}
\hline \multirow{2}{*}{$\mathrm{LL}_{1}$} & $\mathrm{LL}_{2}$ & $\mathrm{HL}_{2}$ \\
\cline { 2 - 3 } & $\mathrm{LH}_{2}$ & $\mathrm{HH}_{2}$ \\
\hline $\mathrm{LH}_{1}$ & \multicolumn{2}{|c|}{$\mathrm{HH}_{1}$} \\
\hline
\end{tabular}

Figure 2. Frame’s 2-level DWT sub-bands. 
Apparently, Matrix S is a triangular matrix; and it is also similar to A by definition of matrices similarities [20]. Accordingly, matrix S has the same multi set of Eigen values in A. Eigen values set is contained in the main diagonal of the $\mathrm{S}$ matrix because $\mathrm{S}$ is triangular. Hence, the diagonal in $\mathrm{S}$ matrix was selected in our technique for watermarking embedding, because of the fact that Eigen values in Schur decomposition are highly stable [22].

\section{The Proposed Technique}

\subsection{Video Frames and Video Watermarking}

The host in any digital watermarking technique is defined as the place where the watermark is embedded. The host can be image, video or audio. A video sequence consists of many frames where each frame can be considered as an image. So image watermarking can be extended to video watermarking. The watermark in the proposed technique is embedded in the frames of a video clip.

\subsection{The Proposed DWT-Schur Technique}

In this paper a blind robust digital video watermarking technique is proposed. The proposed technique applies both DWT and Schur transforms on the digital video where a binary watermark in embedded and distributed in the video frames. The proposed DWT-Schur watermarking algorithm consists of two procedures, the first embeds the watermark into the original video clip, while the other extracts it form the watermarked version of the clip. The two procedures are described in details in the following sections.

\section{Watermark Embedding Procedure}

The proposed DWT-SCHUR Based Watermarking Algorithm begins with embedding the selected binary watermark into the video frames; the embedding procedure is described in details in Table 1.

\section{Table 1. Embedding procedure steps.}

\section{Input Video}

1. Input the video clip V.

2. Divide the video clip into video scenes $\mathrm{Vs}_{\mathrm{i}}$

3. Process the frames of each video scene using DWT and Schur as described in steps 4 - 11.

4. Convert every video frame F from RGB to YUV color matrix format.

\section{Apply 2-level DWT}

5. Compute the 2-level DWT for the Y (luminance) matrix in each frame F. This operation generates seven DWT sub-bands [ $\mathrm{LL}_{1}, \mathrm{LL}_{2}, \mathrm{HL}_{2}$, $\left.\mathrm{LH}_{2}, \mathrm{HH} 2, \mathrm{LH}_{1}, \mathrm{HH}_{1}\right]$.

\section{Apply SCHUR}

6. Apply the Schur operator on the $\mathrm{HL}_{2}$ sub-band (highlighted in Figure 1). The Schur operator decomposes the sub-band's coefficient matrix into two independent matrices:

$$
\mathrm{HL}_{2}=\left(\mathrm{U}_{\mathrm{HL}_{2}} \mathrm{~S}_{\mathrm{HL}_{2}}\right)
$$

\section{Embedding}

7. Rescale the watermark image so that the size of the watermark will match the size of the $\mathrm{HL}_{2}$ sub-band which will be used for embedding. 8. Embed the binary bits of watermark $\mathrm{W}_{\mathrm{Vsi}}$ into the diagonal matrix of $\mathrm{S}_{\mathrm{HL} 2}$ by substituting the watermark bit $\mathrm{W}_{\mathrm{i}}$ with the eighth LSB (Least significant Bit) bit of $\mathrm{S}_{\mathrm{HL}_{2}}(\mathrm{i}, \mathrm{i})$ :

$$
\operatorname{LSB}\left(\mathrm{S}_{\mathrm{HL}_{2}}(\mathrm{i}, \mathrm{i})\right)=\mathrm{W}_{\mathrm{Vsi}}
$$

\section{Video Reconstruction}

9. Apply the inverse Schur operator on the modified $\mathrm{S}_{\mathrm{HL}_{2}}^{\prime}$ matrix to get a modified coefficient matrix $\mathrm{HL}_{2}^{\prime}$. The inverse Schur operation is as follows:

$$
\left(\mathrm{U}_{\mathrm{HL}_{2}} \times \mathrm{S}_{\mathrm{HL}_{2}} \times \mathrm{U}_{\mathrm{HL}_{2}}^{\mathrm{T}}\right)
$$

10. Apply the inverse DWT on the modified coefficient matrix $\mathrm{HL}_{2}^{\prime}$. This operation produces the final watermarked Video frame $\mathrm{F}^{\prime}$.

11. Convert the video frames f' from YUV to RGB color matrix.

12. Reconstruct frames into the final watermarked Video scene $F^{\prime}$.

13. Reconstruct watermarked scenes to get the final watermarked VXM. 
The embedding procedure described previously is depicted in the block diagram shown in Figure 3.

\subsection{Watermark Extraction Procedure}

The Watermark extraction procedure does not need the original video given that the proposed DWT-Schur algorithm is totally blind. Consequently, we can extract the watermark image from the watermarked video frames from the LSBs directly. The watermarking extraction procedure is described in details in Table 2.

The watermarking extraction procedure proposed above is illustrated in the block diagram shown in Figure 4 .

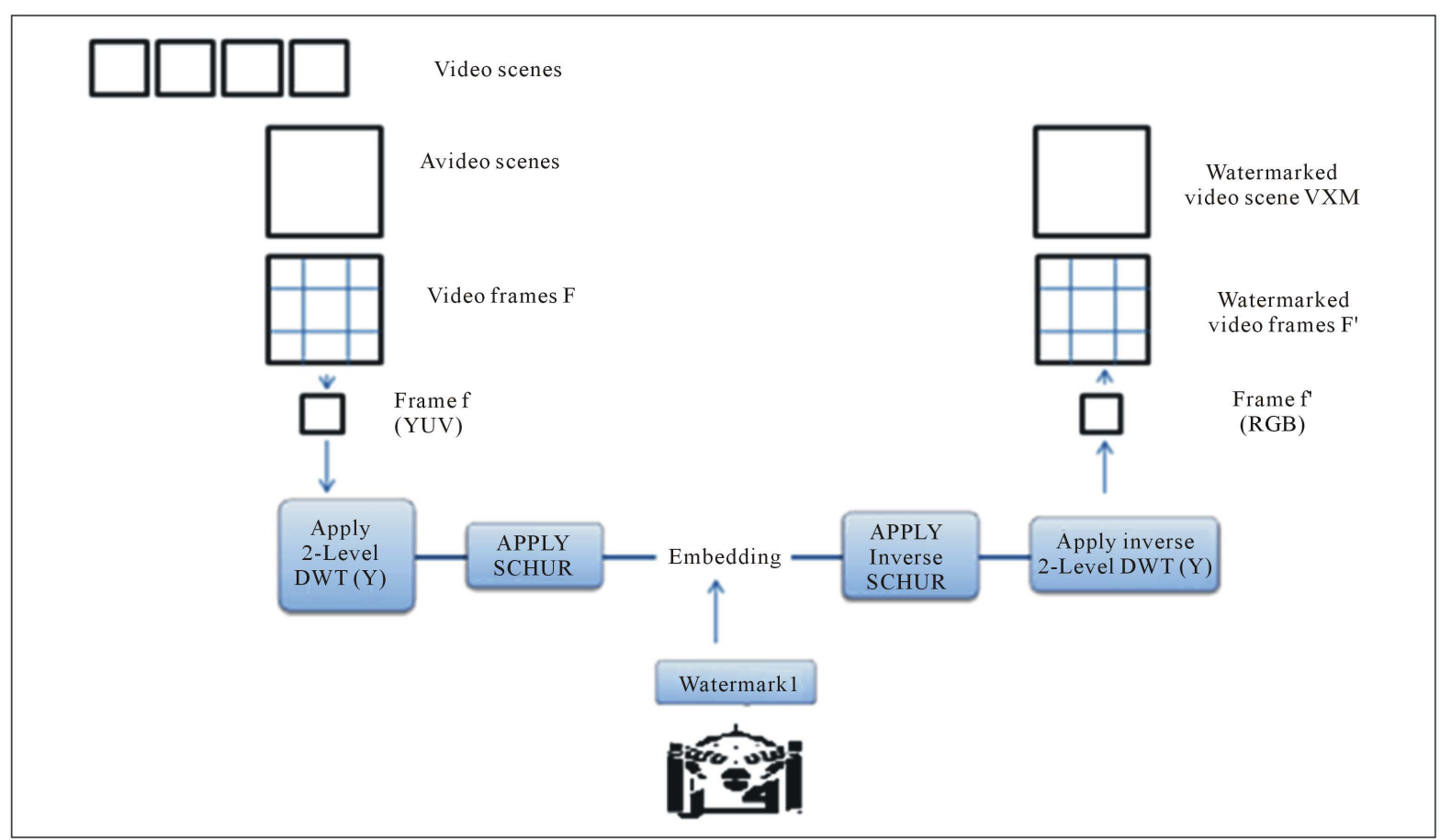

Figure 3. The watrermark embedding procedure.

Table 2. Extraction procedure steps.

\section{Input Video}

1. Input the watermarked Video clip $\mathrm{V}^{\prime}$.

2. Divide the watermarked Video clip $V^{\prime}$ into watermarked scenes Vsi'.

3. Process the watermarked frames of each watermarked video scene using DWT and Schur as described in steps 4 - 7.

4. Convert the video frame $F^{\prime}$ from RGB color matrix to YUV.

\section{Apply 2-level DWT}

5. Compute the 2-level DWT for the frame $\mathrm{F}^{\prime}$. Let the seven sub-bands produced after this process be: [wLL $\mathrm{wLL}_{2}, \mathrm{wHL}_{2}, \mathrm{wLH}_{2}, \mathrm{wHH}_{2}$, $\left.\mathrm{wLH}_{1}, \mathrm{wHH}_{1}\right]$.

\section{Apply SCHUR}

6. Apply the Schur operator on the $\mathrm{wHL}_{2}$ sub-band. The Schur operator decomposes the sub-band's coefficient matrix into two independent matrices:

$$
\mathrm{W}_{\mathrm{HL}_{2}}=\mathrm{U}_{\mathrm{WHL}_{2}} \mathrm{~S}_{\mathrm{WHL}_{2}}
$$

Extraction

7. Extract the embedded watermark from the diagonal matrix $S_{\text {wнL2 }}$ as follows:

$$
\mathrm{W}_{\mathrm{Vsi}}(\mathrm{i})=\operatorname{LSB}\left(\mathrm{S}_{\mathrm{HL}_{2}}(\mathrm{i}, \mathrm{i})\right)
$$

\section{Video Reconstruction}

8. Construct the image watermark WVsi by cascading all watermark bits extracted from all frames.

9. Repeat the same procedure for all video scenes.

10. The watermarking extraction procedure proposed above is illustrated in the block diagram shown in Figure 3. 


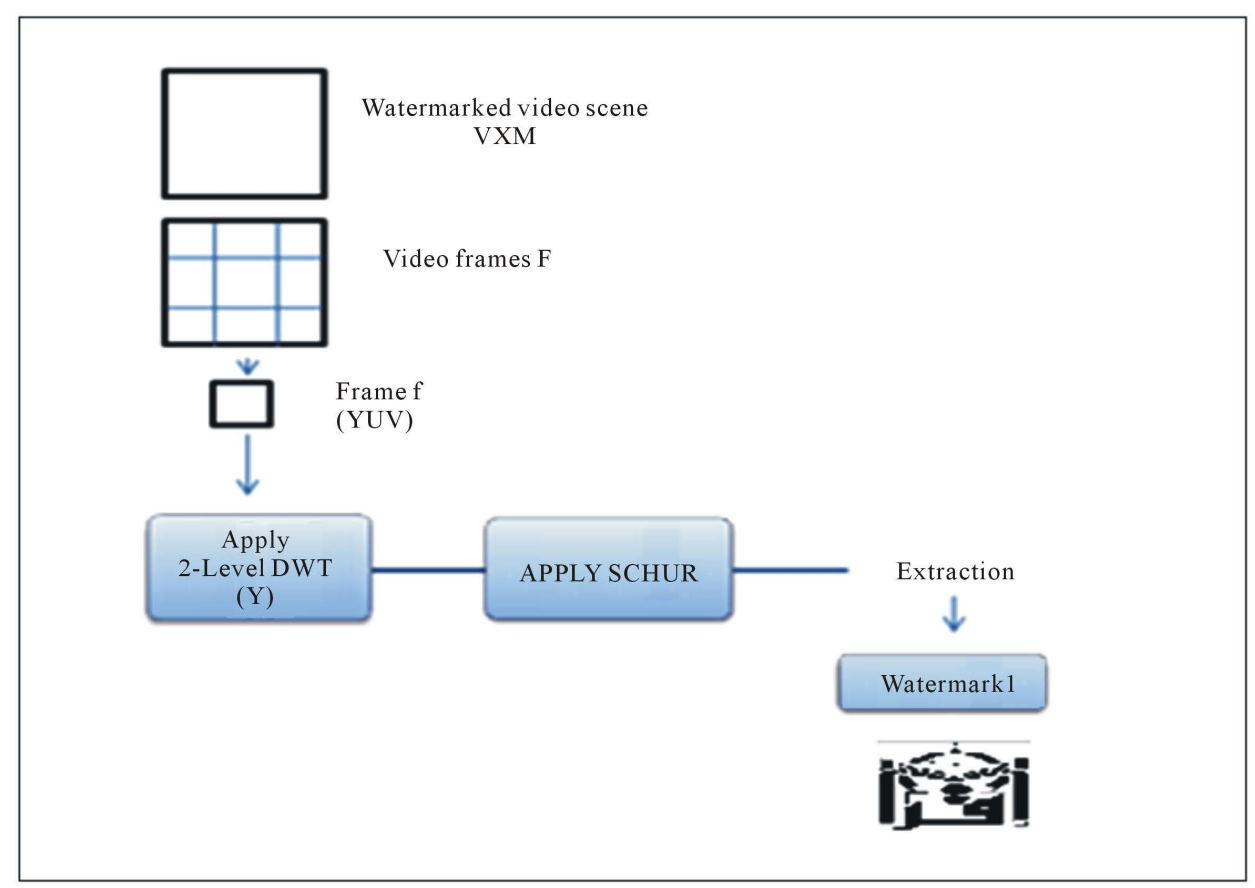

Figure 4. The watermark extraction procedure.

\section{Experimental Results}

In this section we investigate the robustness of the proposed DWT-Schur technique against different attacks. The cover video clip used for testing has four scenes with 438, 599, 744 and 821 frames respectively. The size for each frame is $352 \times 352$ pixels. A snapshot from each scene is shown in Figure 5 . The watermark is a binary text image with size $363 \times 256$ as shown in Figure 6 . The use of text messages makes it easy to test the robustness. Where we simply read the extracted message and compare it with the original watermark.

To measure the level of robustness and the similarity between the original watermark $\mathrm{W}$ and the extracted watermarks W' the correlation shown in the Equation (7) is used. The correlation may take values between 0 and 1 . Where 0 indicates that there is no similarity and 1 indicates that both watermarks are same.

$$
\operatorname{Corr}\left(\mathrm{W}, \mathrm{W}^{\prime}\right)=\frac{\mathrm{x}^{*} \mathrm{x}^{\mathrm{t}}}{\sqrt{\mathrm{XX}^{\mathrm{T}}}}
$$

In the Normal Test the proposed technique shows a high imperceptibility where. No visual degradation was noticed in the video clip after embedding the watermark and the similarity between the embedded and extracted watermark are completely similar as shown in Figure 7. The average PSNR for the all watermarked frames was 57.3118. At this PSNR value no quality degradation in the watermarked video was perceived the PSNR is shown in Equation (8).

$$
\operatorname{PSNR}=10 \log 10(255 / \sqrt{\mathrm{MSE}})
$$

The robustness for the proposed technique is measured against two types of attacks: standard and frame attacks. Standard attacks include compression, rotation, Gaussian noise, salt \& pepper noise, among many others [2]. The video attacks are frames swapping, averaging and dropping. For both types of attacks we measured the similarity between the original and extracted watermarks using the correlation.

Salt \& Pepper and Gaussian Noise Addition Attacks The robustness of the proposed technique against salt \& pepper and Gaussian noise is acceptable since the watermark is still distinguished and the correlation results are somehow high but the lowest compared with the other attacks which is reasonable since the noise addition attacks affects each pixel in the watermarked frame (the results are shown in Table 3). 
Table 3. The results after applying the noise addition attacks.

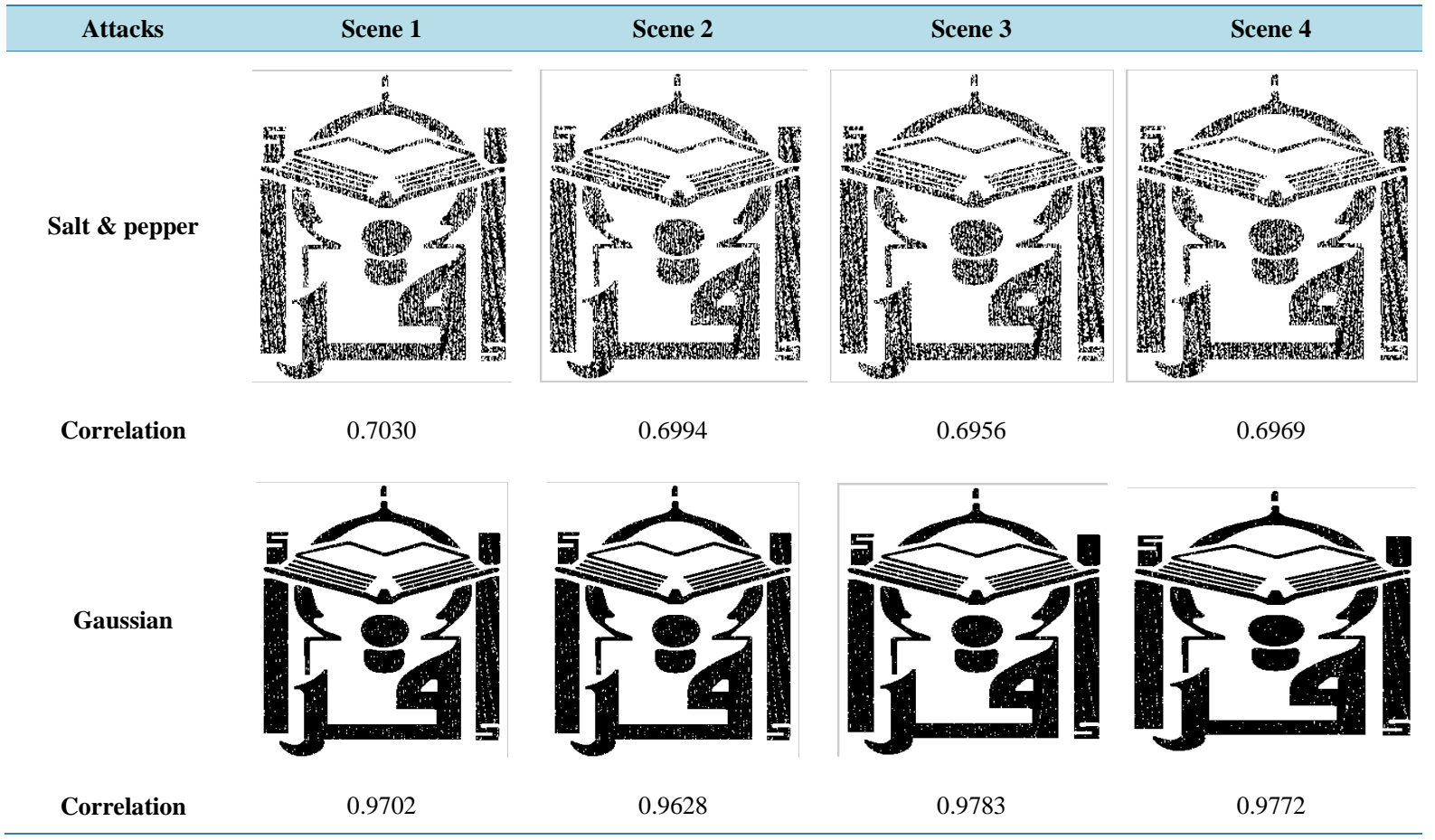

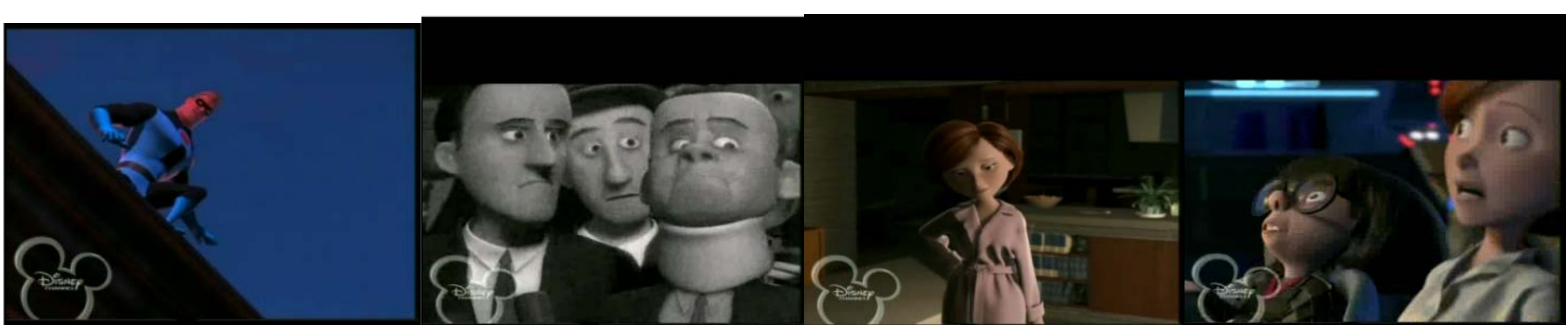

Figure 5. Snapshots from the video scenes.

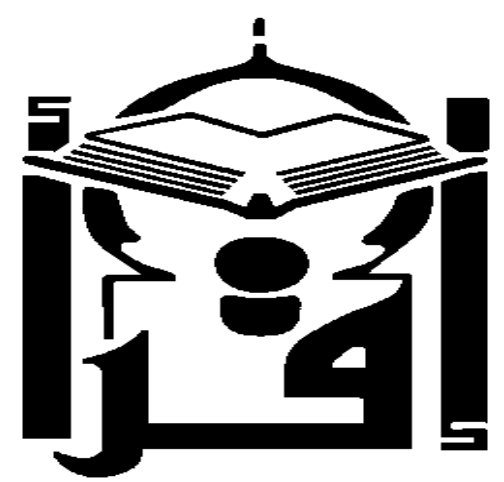

Figure 6. The original watermark.

Rotation Attacks for the rotation attacks we rotated the watermarked image by three different angels 30, 90 and 180 degrees. The proposed techniques shows high robustness against this attack as you see in Table 4 the watermarks are almost the same as the original with high values of correlation. Hence, the diagonal in $\mathrm{S}$ matrix was selected in our technique for watermarking embedding as we discussed before and this is because of the fact that Eigen values in Schur decomposition are highly stable and increase the robustness. 
Table 4. The results after applying the rotation attacks.

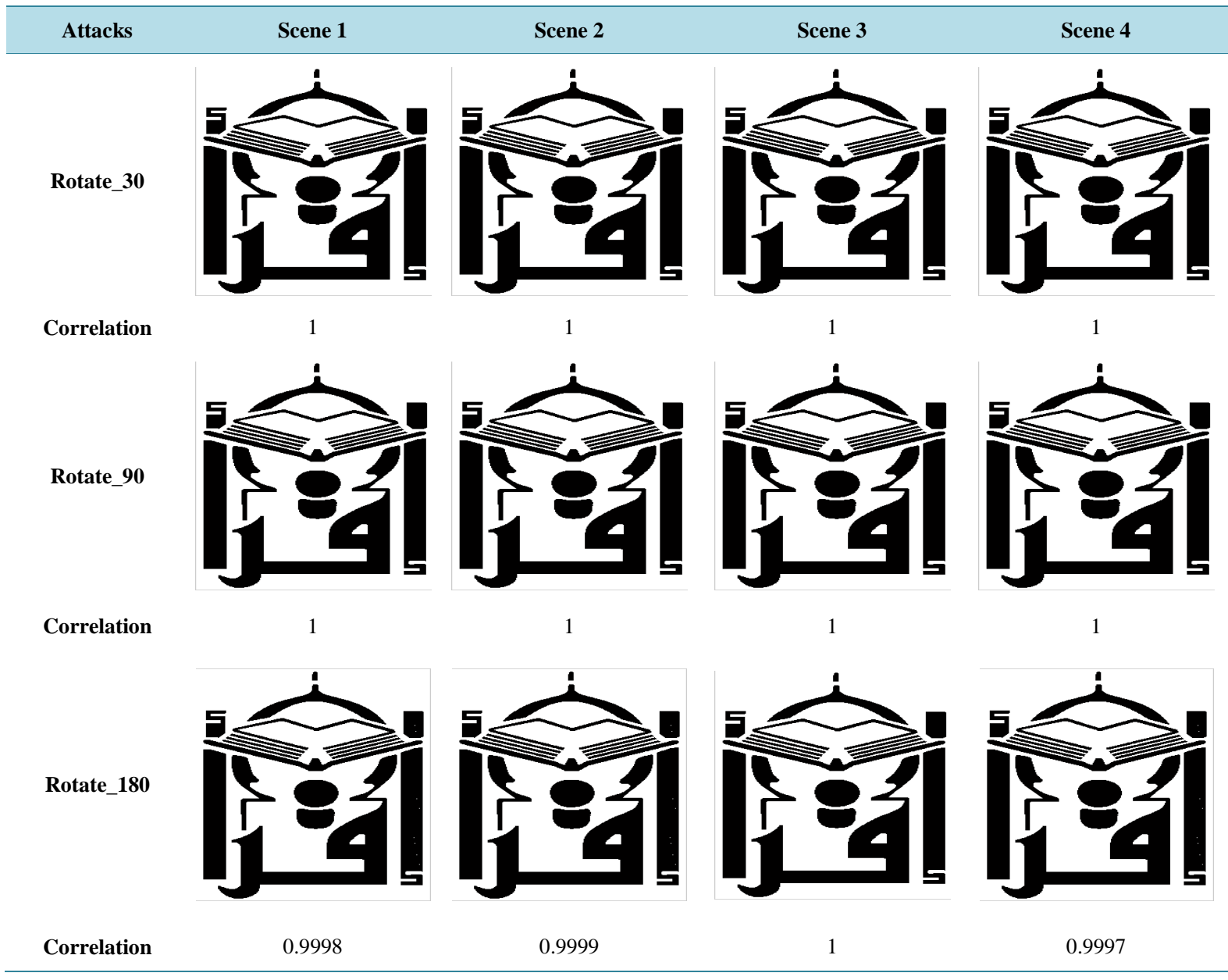

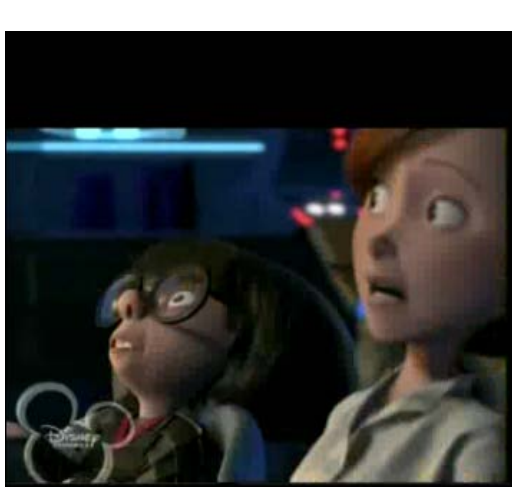

Watermarked Video

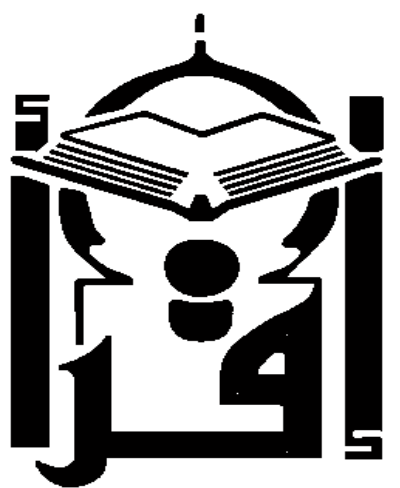

Extracted watermark from Normal Test

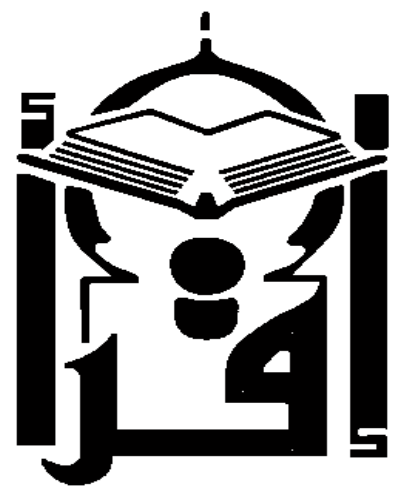

Original watermark

Figure 7. The watermarked and the extracted watermark in the normal test.

Video Attacks for video attacks we test the robustness for the watermarked scenes by applying frames swaping attacks where the fourth and the tenth frames in each watermarked scene are swapped. Then frame averaging video attacks and finally the frame dropping video attacks where we dropped $90 \%$ from the watermarked scene and as you see in Table 5 the results are reflected in the extracted watermark where approximately we see 10\% of the watermark and the remaining is dropped with the video. This is because the watermark is distributed in all the frames in each video scene. 
Table 5. The results after applying the video attacks.

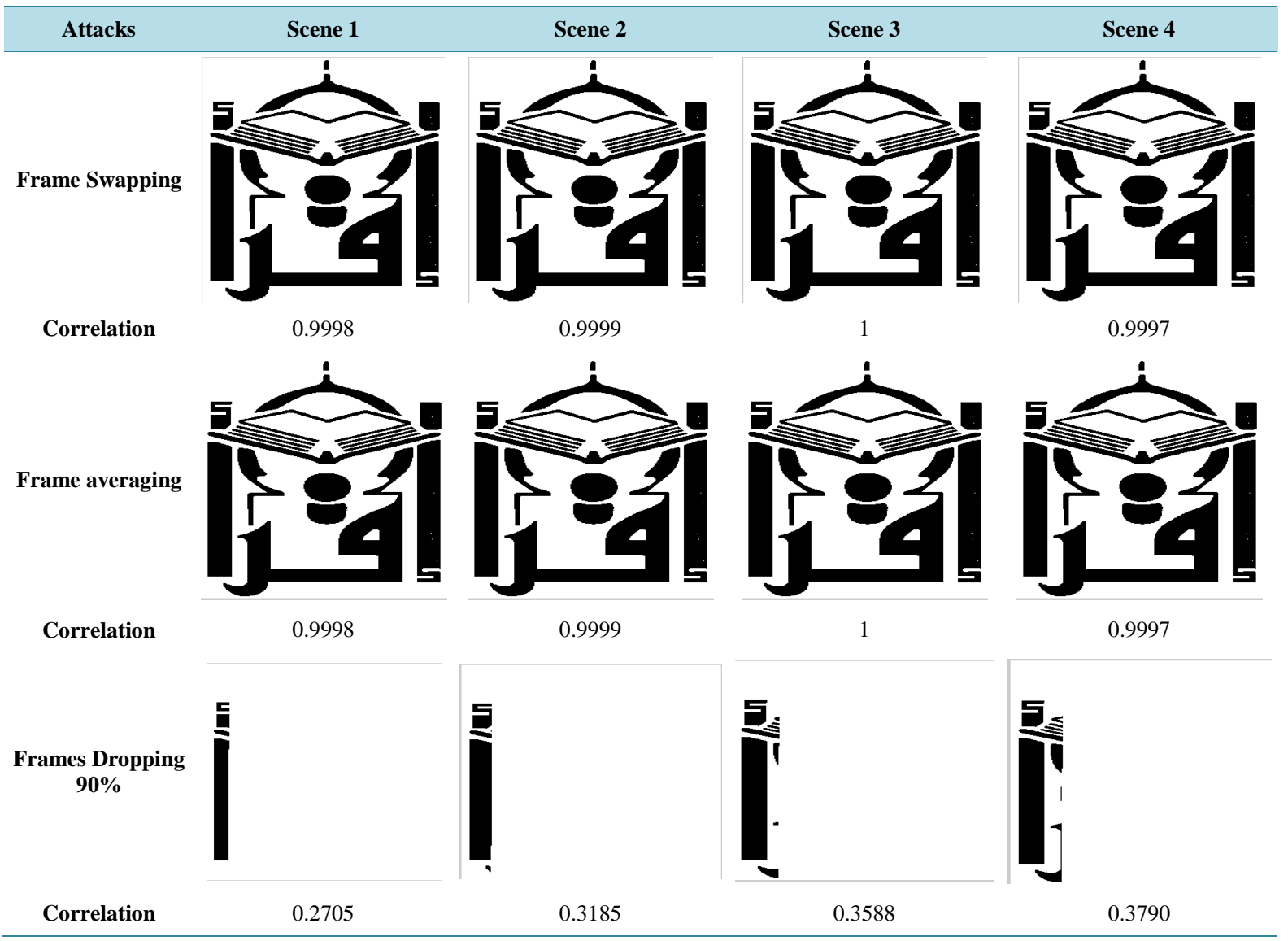

\section{Conclusion}

This paper presented a new technique for blind digital video watermarking. The proposed technique made use of two powerful mathematical transforms: Discrete wavelet transforms (DWT) and Schur decomposition. Experimental results demonstrated the imperceptibility, blindness and robustness of the proposed technique. The watermark was successfully extracted without the existence of the original video and no visual distortion appears in the watermarked video. The technique proved its robustness against a set of standard and video attacks, and succeeded in extracting the watermark with minimum distortion. Using Schur decomposition improved the efficiency of the technique due to the small number of computations compared to other similar mathematical decompositions.

\section{References}

[1] Khatib, T., Haj, A., Rajab, L. and Mohammed, H. (2008) A Robust Video Watermarking Algorithm. Journal of Computer Science, 4, 910-915. http://dx.doi.org/10.3844/jcssp.2008.910.915

[2] Voloshynovskiy, S., Pereira, S. and Pun, T. (2001) Attacks on Digital Watermarks: Classification, Estimation-Based Attacks, and Benchmarks. Communications Magazine, 39, 118-126. http://dx.doi.org/10.1109/35.940053

[3] Gunjal, B.L. and Manthalkar, R.R. (2010) An Overview of Transform Domain Robust Digital Image Watermarking Algorithms. Journal of Emerging Trends in Computing and Information Sciences, 2, 37-42.

[4] Lancini, R., Mapelli, F. and Tubaro, S. (2002) A Robust Video Watermarking Technique in the Spatial Domain. Video/Image Processing and Multimedia Communications 4th EURASIP-IEEE Region 8 International Symposium on VIPromCom, 2002, 251-256. http://dx.doi.org/10.1109/VIPROM.2002.1026664

[5] Hussein, J.A. (2010) Spatial Domain Watermarking Scheme for Colored Images Based on Log-Average Luminance. CoRR, abs/1001.3496. 
[6] Mondal, M. and Barik, D. (2012) Spatial Domain Robust Watermarking Scheme for Color Image. International Journal of Advanced Computer Science, 2, 24-27.

[7] Mostafa, S.A., Tolba, A.S., Abdelkader, F.M. and Elhindy, H.M. (2009) Video Watermarking Scheme Based on Principal Component Analysis and Wavelet Transform. International Journal of Computer Science and Network Security, 9, 45-52.

[8] Al-Taweel, S.A., Sumari, P., Alomari, S.A. and Husain, A.J. (2009) Digital Video Watermarking in the Discrete Cosine Transform Domain. Journal of Computer Science, 5, 536-543. http://dx.doi.org/10.3844/jcssp.2009.536.543

[9] Sathik, M. and Sujatha, S. (2012) A Novel DWT Based Invisible Watermarking Technique for Digital Images. International Arab Journal of e-Technology, 2, 167-172.

[10] Hernández, M.C., Miyatake, M.N. and Meana, H.P. (2005) Analysis of a DFT-Based Watermarking Algorithm. 2nd International Conference on Electrical and Electronics Engineering, 7-9 September 2005, 44-47. http://dx.doi.org/10.1109/ICEEE.2005.1529569

[11] Rajab, L., Al-Khatib, T. and Al-Haj, A. (2009) Video Watermarking Algorithms Using the SVD Transform. European Journal of Scientific Research, 30, 389-401.

[12] Rajab, L., Al-Khatib, T. and Al-Haj, A. (2008) Hybrid DWT-SVD Video Watermarking. Proceedings of the International Conference on Innovations in Information Technology, Al Ain, 16-18 December 2008, 588-592. http://dx.doi.org/10.1109/INNOVATIONS.2008.4781696

[13] Meenakshi, K., Rao, C.S. and Prasad, K.S. (2014) A Fast and Robust Hybrid Watermarking Scheme Based on Schur and Svd Transform. International Journal of Research in Engineering and Technology, 3, 7-11.

[14] Panahi, N., Amirani, M., Behnia, S. and Ayubi, P. (2013) A New Colour Image Watermarking Scheme Using Cellular Automata Transform and Schur Decomposition. Proceedings of the 21st Iranian Conference on Electrical Engineering (ICEE), Mashhad, 14-16 May 2013, 1-5. http://dx.doi.org/10.1109/IranianCEE.2013.6599623

[15] Seddik, H., Sayadi, M. and Fnaiech, F. (2009) A New Blind Image Watermarking Method Based on Shur Transformation. Proceedings of the 35th Annual Conference of IEEE Industrial Electronics, Porto, 3-5 November 2009, 19671972.

[16] Mohan, B.C. and Swamy, K.V. (2010) On the Use of Schur Decomposition for Copyright Protection of Digital Images. International Journal of Computer and Electrical Engineering, 2, 781-787. http://dx.doi.org/10.7763/IJCEE.2010.V2.228

[17] Xia, X.G., Boncelet, C.G. and Arce, G.R. (1997) A Multiresolution Watermark for Digital Images. Proceedings of the International Conference on Image Processing, Santa Barbara, 26-29 October 1997, 548-551. http://dx.doi.org/10.1109/ICIP.1997.647971

[18] Hong, W. and Hang, M. (2006) Robust Digital Watermarking Scheme for Copy Right Protection. IEEE Transactions on Signal Processing, 12, 1-8.

[19] Kundur, D. and Hatzinakos, D. (1998) Digital Watermarking Using Multiresolution Wavelet Decomposition. Proceedings of the 1998 IEEE International Conference on Acoustics, Speech and Signal Processing, Seattle, 12-15 May 1998, 2969-2972. http://dx.doi.org/10.1109/ICASSP.1998.678149

[20] Golub, G.H. and Van Loan, C.F. (1989) Matrix Computations. Johns Hopkins University Press, Baltimore.

[21] Mohammad, A.A. (2012) A New Digital Image Watermarking Scheme Based on Schur Decomposition. Multimedia Tools and Applications, 59, 851-883. http://dx.doi.org/10.1007/s11042-011-0772-7

[22] Mohan, B.C., Swamy, K.V. and Kumar, S.S. (2011) A Comparative Performance Evaluation of SVD and Schur Decompositions for Image Watermarking. IJCA Proceedings on International Conference on VLSI, Communications and Instrumentation (ICVCI), 14, 25-29. 